\title{
Longitudinal three-dimensional visualisation of autoimmune diabetes by functional optical coherence imaging
}

\author{
Corinne Berclaz ${ }^{1}$ • Anja Schmidt-Christensen ${ }^{2}$ - Daniel Szlag ${ }^{1,3}$ • Jerome Extermann ${ }^{1,4}$. \\ Lisbeth Hansen $^{2}$ • Arno Bouwens ${ }^{1}$ - Martin Villiger ${ }^{1}$. Joan Goulley ${ }^{5}$. Frans Schuit $^{6}$. \\ Anne Grapin-Botton ${ }^{7}$. Theo Lasser ${ }^{1} \cdot$ Dan Holmberg $^{2}$
}

Received: 8 September 2015 / Accepted: 30 October 2015 / Published online: 27 November 2015

(C) Springer-Verlag Berlin Heidelberg 2015

\begin{abstract}
Aims/hypothesis It is generally accepted that structural and functional quantitative imaging of individual islets would be beneficial to elucidate the pathogenesis of type 1 diabetes. We here introduce functional optical coherence imaging (FOCI) for fast, label-free monitoring of beta cell destruction and associated alterations of islet vascularisation.

Methods NOD mouse and human islets transplanted into the anterior chamber of the eye (ACE) were imaged with FOCI, in which the optical contrast of FOCI is based on intrinsic variations of the index of refraction resulting in a faster tomographic acquisition. In addition, the phase sensitivity allows simultaneous label-free acquisition of vascularisation.

Results We demonstrate that FOCI allows longitudinal quantification of progressive autoimmune insulitis, including the
\end{abstract}

three-dimensional quantification of beta cell volume, inflammation and vascularisation. The substantially increased backscattering of islets is dominated by the insulin-zinc nanocrystals in the beta cell granules. This translates into a high specificity for the functional beta cell volume of islets. Applying FOCI to a spontaneous mouse model of type 1 diabetes, we quantify the modifications of the pancreatic microvasculature accompanying the progression of diabetes and reveal a strong correlation between increasing insulitis and density of the vascular network of the islet.

Conclusions/interpretation FOCI provides a novel imaging technique for investigating functional and structural diabetes-induced alterations of the islets. The label-free detection of beta cell volume and infiltration together with vascularisation offers a unique extension to study ACE-transplanted

Corinne Berclaz, Anja Schmidt-Christensen and Daniel Szlag contributed equally to this study.

Electronic supplementary material The online version of this article (doi:10.1007/s00125-015-3819-x) contains peer-reviewed but unedited supplementary material, which is available to authorised users.

Theo Lasser

theo.lasser@epfl.ch

Dan Holmberg

dan.holmberg@med.lu.se

1 Laboratoire d'Optique Biomédicale,

Ecole Polytechnique Fédérale de Lausanne,

CH1015 Lausanne, Switzerland

2 EMV - Immunology, Lund University, BMC, D14, 22184 Lund, Sweden

3 Institute of Physics, Faculty of Physics, Astronomy and Informatics, Nicolaus Copernicus University, Torun, Poland
Hepia, University of Applied Science of Western Switzerland, Genève, Switzerland

5 Swiss Institute for Experimental Cancer Research, Ecole Polytechnique Fédérale de Lausanne, Lausanne, Switzerland

6 Gene Expression Unit, Department of Molecular and Cellular Medicine, Katholieke Universiteit Leuven, Leuven, Belgium

DanStem, University of Copenhagen, Copenhagen, Denmark 
human islets. These results are contributing to a deeper understanding of human islet transplant rejection and label-free in vivo monitoring of drug efficacy.

Keywords 3D visualisation $\cdot$ Beta cell volume $\cdot$ Human islets · Inflammation $\cdot$ Label-free $\cdot$ Longitudinal $\cdot$ NOD mouse $\cdot$ Non-invasive $\cdot \mathrm{OCM} \cdot$ Quantification $\cdot$ Vasculature

$\begin{array}{ll}\text { Abbreviations } & \\ \text { 3D } & \text { Three-dimensional } \\ \text { ACE } & \text { Anterior chamber of the eye } \\ \text { B6 } & \text { C57BL/6 } \\ \text { CT } & \text { Computed tomography } \\ \text { dfOCM } & \text { Dark-field OCM } \\ \text { FOCI } & \text { Functional optical coherence imaging } \\ \text { FOXP3 } & \text { Forkhead box P3 } \\ \text { GFP } & \text { Green fluorescent protein } \\ \text { HEV } & \text { High endothelial venule } \\ \text { IHC } & \text { Immunohistochemistry } \\ \text { KO } & \text { Knockout } \\ \text { MAdCAM-1 } & \text { Mucosal addressin cell adhesion molecule 1 } \\ \text { OCM } & \text { Optical coherence microscopy } \\ \text { OPT } & \text { Optical projection tomography } \\ \text { xfOCM } & \text { Extended-focus OCM }\end{array}$

\section{Introduction}

Diabetes mellitus develops as a functional impairment in insulin production, sometimes in association with insulin resistance. In both major types of diabetes mellitus, the progressive dysfunction of the beta cell causes the disease development. While type 1 diabetes is the result of an autoimmune attack on beta cells, type 2 diabetes is considered to be driven by metabolic factors associated with sedentary lifestyle and obesity, albeit with accumulating evidence of low-grade inflammation. Modifications of the pancreatic microvasculature are likely to accompany the progression of both types of diabetes. Alterations in vascular variables, such as transient vasoconstriction, vasodilation, increased blood flow and vascular leakage, are necessary preludes to inflammation as they orchestrate the influx of diverse cell types and affect local homeostasis [1-6]. To fully appreciate how these events contribute to the pathogenesis, improved methods allowing longitudinal, highresolution monitoring of vascularisation and affected tissues close to the cellular level during its natural progression are warranted and constitute the key objectives of this study.

To date, longitudinal non-invasive, intra-vital imaging of the pancreas has been mainly restricted to non-optical imaging modalities such as MRI and computed tomography (CT). While the medical potential of these modalities is indisputable, they are limited by a restricted set of reagents targeting specific tissues and by a relatively low spatial resolution $[4,7,8]$. Optical microscopy on the other hand is mainly limited to preclinical studies because of a reduced penetration depth [9-12]. Optical projection tomography (OPT) has been used to image the adult mouse pancreas and retrieve the three-dimensional (3D) and undistorted structure of the tissue [13]. While OPT can quantify inflammation at different stages of disease $[14,15]$, it is limited by being an ex vivo technique that requires sample fixation and immunolabelling.

In accordance with these objectives, we opted for a noninvasive imaging method, extended-focus optical coherence microscopy (xfOCM) [16], which allows label-free longitudinal visualisation and quantification of pancreatic ducts, blood vessels and individual islets of Langerhans ex vivo and in vivo. The technique requires only a two-dimensional scan to obtain a 3D image. This intrinsic multiplex advantage results in a fast parallel acquisition of entire depth profiles without depth scanning, with an in vivo penetration depth of $\sim 300 \mu \mathrm{m}$ in the pancreas. However, optical pancreas imaging requires laparotomy, restricting this technique to transversal studies [17-19]. Recently, the anterior chamber of the eye (ACE) has been used to study transplanted pancreatic islets [20-22]. Engrafted on the iris, islets could now be repeatedly imaged using the mouse eye as a natural body window. Using this approach, we elucidate important structural and functional alterations induced during the progression of autoimmune diabetes.

\section{Methods}

Animals Mice of genotype C57BL/6 (B6). Rag2 $^{-/-}$were backcrossed with NOD mice for the generation of NOD. $\operatorname{Rag} 2^{-1-}$ mice, as previously described [23]. NOD.Foxp3-green fluorescent protein (GFP) mice were generated by speed-congenic backcrossing of the B6.Foxp3-GFP (Jax stock number 006772) with NOD mice, as previously described [21]. BALB/c mice were purchased from Taconic (Ejby, Denmark). All animals were bred and maintained in a specific pathogen-free environment at the animal facilities at Lund University. Guinea pigs were purchased from Harlan Laboratories (Indianapolis, IN, USA). Mice with ZnT8 (also known as Slc30a8) knockout (KO) and littermates were generated as previously described [24]. The Ethics Committee at Lund University and the Swiss cantonal veterinary authorities approved all experimental animal procedures. Experiments were performed after mice were genotyped, without randomisation or blinding. No data were specifically included or excluded.

Pancreatic islet isolation, culture and ACE transplantation Mouse pancreatic islets were isolated according to the 
protocol previously described [25]. Between 10 and 50 cultured islets were transplanted per eye, as previously described [21].

For experiments, including the study of healthy control islet grafts, 6-8-week old BALB/c or B6.Foxp3-GFP recipient mice were $\mathrm{ACE}$ transplanted with islets from $\mathrm{BALB} / \mathrm{c}$ or B6.Rag $2^{-1-}$ donors, respectively. For inflamed islet grafts, 10week-old NOD.Foxp3-GFP recipient mice were ACE transplanted with islets from NOD.Rag $2^{-/-}$donors. Human islets from non-diabetic cadaver donors were provided by the Nordic Islet Transplantation Program (www.nordicislets. org), coordinated by O. Korsgren, Uppsala University, and were transplanted into 6-8-week-old female NOD.Rag $2^{-/-}$ mice (see the electronic supplementary materials [ESM] Methods for details). The study was approved by the Ethics Committee at Lund University.

Functional optical coherence imaging Mouse head and eyeball were restrained as previously described [20] and anaesthesia was maintained at $1 \%$ isoflurane. Functional optical coherence imaging (FOCI) integrates the XfOCM instrument equipped with a Zeiss Neofluar objective $\times 10$; NA 0.3, Carl Zeiss (Jena, Germany) with an improved acquisition (customised optical coherence microscopy [OCM] spectrometer) and optimised scanning modality for longitudinal functional imaging. A fast processing unit allows real-time monitoring (structure and blood flow), with a fully equipped platform for small-animal imaging. Details of the xfOCM instrument, data acquisition, scanning protocols and analysis are given in the ESM Methods.

Immunohistochemistry Graft-bearing eyes or pancreas were isolated from mice after perfusion with $4 \%$ paraformaldehyde/ PBS, cryosectioned parallel to the iris (section thickness $8-10 \mu \mathrm{m})$ and subjected to immunohistochemical staining as previously described [21] (see ESM Methods).

Ex vivo imaging with dark-field optical coherence microscopy Cryosections were mounted with DABCO mounting medium and imaged using the dual system combining darkfield OCM (dfOCM) [26] with confocal fluorescence (ESM Fig. 1) (see ESM Methods).

Statistics Groups were compared by the Mann-Whitney test or unpaired Student's $t$ test, as indicated in the respective figure legend, and data are expressed as means \pm SEM.

\section{Results}

Beta cell specificity in OCM The optical contrast in XfOCM is based on the scattering characteristics of the investigated tissue. As we have previously shown, xfOCM imaging can be used to identify pancreatic islets in surgically exposed pancreases of live animals; the strong light scattering observed correlates strongly with insulin-producing beta cells [17]. As OCM is sensitive to changes in refractive index and because pancreatic beta cells have a high zinc content [27], we hypothesised that the origin of the strong OCM signal is dominantly caused by the zinc-insulin crystals in the pancreatic beta cells. To test this hypothesis, thick pancreas sections of ZnT8-KO mice, which lack zinc-insulin crystals in the secretory granules of beta cells [24, 27], were subjected to immunohistochemistry (IHC) using antiinsulin antibodies and analysed simultaneously by OCM and confocal fluorescence microscopy. In order to suppress the specular reflection from the cover slip, we used a darkfield OCM (dfOCM) configuration combined with a confocal fluorescence channel (ESM Fig. 1). Figure 1 shows transverse virtual sections at the selected depth position of this dfOCM image stack and the corresponding fluorescence images. While control pancreases showed a good correlation between dfOCM and insulin staining (Fig. 1a), the dfOCM signal in the ZnT8-KO mouse pancreases was significantly diminished (Fig. 1b, d), indicating that zinc within beta cells contributes to the OCM signal. In addition, we imaged the pancreases of guinea pigs, as they have a divergent insulin unable to form the hexameric crystals with zinc [28]. Therefore, beta cells in guinea pigs contain both insulin and zinc, but do not store insulin as zincinsulin crystals. Guinea pig beta cells did not show a strong scattering in dfOCM images (Fig. 1c, d). While it is likely that additional, unidentified nanocrystals contribute to the islet scattering, these findings underline that this label-free approach provides a high specific contrast for the functional beta cell volume.

Longitudinal quantification of beta cell volume and islet vascularisation using FOCI Having established that the xfOCM signal specifically reflects the beta cell volume, we next applied this technique in longitudinal studies. While longitudinal imaging of the pancreas in situ is feasible, the necessary laparotomy does not allow the relocation of the same islet at successive imaging sessions. To overcome this, we combined xfOCM with longitudinal imaging of islets transplanted into the ACE of recipient mice to establish FOCI (ESM Figs 2 and 3). Isolated syngeneic islets were transplanted into the ACE of healthy mice and repeatedly imaged by FOCI. Using this approach, we demonstrated how the islets were growing during the course of the study (Fig. 2a-e). These data extend previous reports based on two-photon microscopy showing that, after an initial phase of vascularisation, syngeneic islets transplanted into the ACE are sustained and function over several months $[20,25]$. 

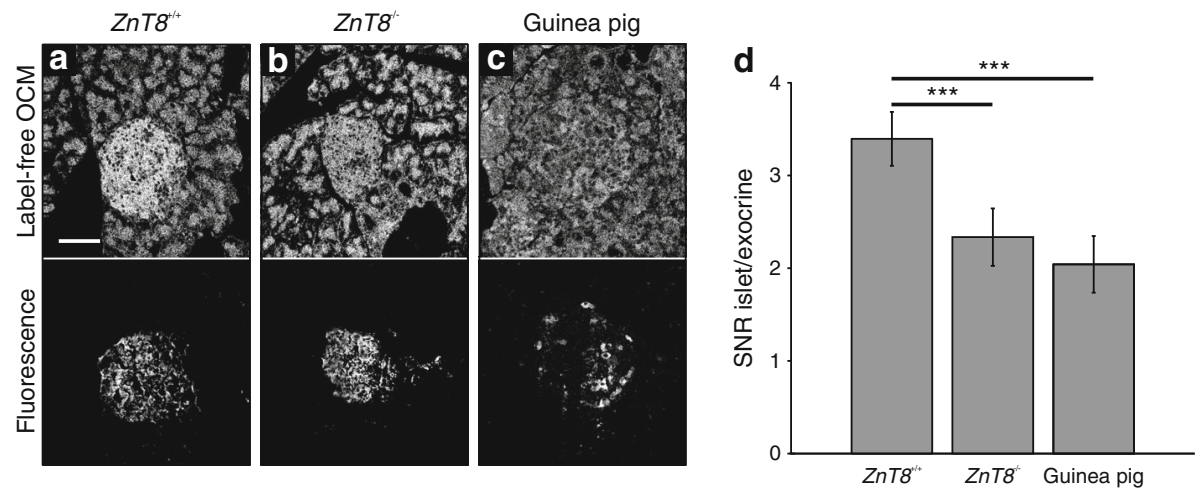

Fig. 1 Ex vivo dfOCM imaging of pancreas and beta cell specificity of the OCM signal. Pancreas sections of $Z n T 8^{+/+}$controls (a), $Z n T 8^{-/-}$mice (b) or guinea pigs (c) were analysed by IHC using anti-insulin $(\mathbf{a}, \mathbf{b})$ or anti-glucagon (c) antibodies. The sections were imaged by dfOCM combined with a confocal fluorescent channel. (d) The signal-to-noise ratio between the islets and the exocrine pancreas was quantified $(n=50-60$ islets per group). The outline of islets was defined by the structural OCM signal and confirmed by IHC (a-c). Scale bar: $50 \mu \mathrm{m} .{ }^{* * *} p<0.001$, Mann-Whitney. SNR, signal-to-noise ratio

varied between three and five and up to 15 islets out of approximately 20 injected islets. Successfully engrafting islets displayed evidence of beginning re-vascularisation within $2-3$ days and become fully re-vascularised $2-3$ weeks later. In these islets, no signs of rejection could be detected for up to 25 weeks. As illustrated in Fig. 3 and as suggested in a previous report [29], transplanted islets were found to be functional and secreting insulin after ACE transplantation. The anatomical organisation of the transplanted islets was intact, with beta cells scattered between alpha and delta cells and distributed over the entire islet volume (Fig 3a). This wellestablished difference from the anatomical organisation of rodent islets is also illustrated in the non-homogeneous tissue structure revealed in the OCM image of the human islet (Fig. 3b). obtained, the successful engraftment of individual islets
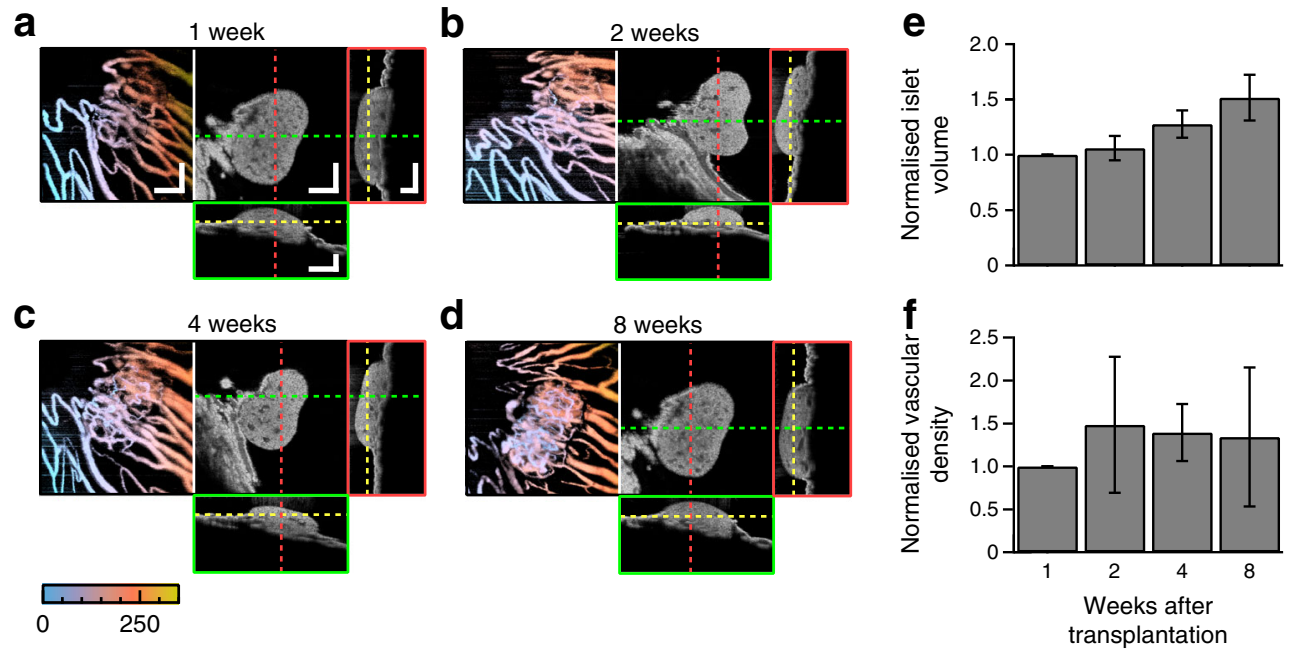

Fig. 2 Longitudinal imaging of ACE-transplanted islets by FOCI and quantification of beta cell volume and islet vascularisation in the healthy state. Islets, transplanted into the ACE of BALB/c control mice $(n=4)$, were imaged by FOCI at the indicated time points and analysed for both structure and dynamic blood flow variables in 3D. (a-d) Maximum projection of the vascularisation and orthogonal view of the islet structure. (e) Islet volume at different time points normalised to the first time point. (f) Islet vascular density (total vessel volume/total islet volume) normalised to the first time point. For each time point, 5-12 islets were analysed. Scale bar: $100 \mu \mathrm{m}$, colour bar indicates depth position in $\mu \mathrm{m}$ 

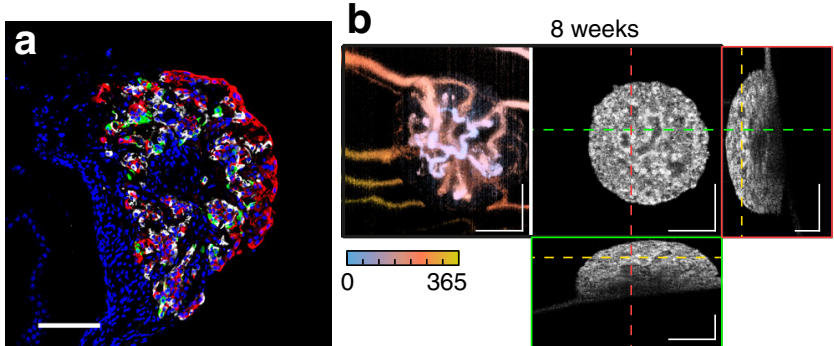

Fig. 3 Imaging of ACE-transplanted human islets by FOCI. Human islets transplanted into the ACE of NOD.Rag2 ${ }^{-/}$mice ( $n=14$ mice), were imaged repeatedly for up to 25 weeks post transplantation. Shown is a representative imaging session by FOCI at 8 weeks post transplantation. (a) Cryosection of the graft-bearing eye at 7 weeks post transplantation stained with antibodies specific for insulin (red), glucagon (white) and somatostatin (green). Nuclei are visualised by DAPI (blue). (b) Maximum projection of the vascularisation and orthogonal view of the islet structure. Colour bar indicates depth position in $\mu \mathrm{m}$. Scale bar: $100 \mu \mathrm{m}$

FOCI detects inflammation in pancreatic islets under autoimmune attack We next applied FOCI to follow the progressive autoimmune destruction of beta cells in the NOD mouse model for type 1 diabetes. To determine if the cells infiltrating the ACE-transplanted islets could be visualised in a label-free manner using FOCI, we next analysed 14-weekold non-diabetic NOD mice transplanted with syngeneic islets 4 weeks prior to analysis.

As reported previously [21] and shown in Fig. 4, NOD reporter mice can be used to visualise specific inflammatory cellular populations infiltrating the pancreatic islet. To interpret the altered OCM signal in vivo during the longitudinal study, we used NOD.Foxp3-GFP reporter mice to visualise the recruitment of forkhead box $\mathrm{P} 3$ (FOXP3) ${ }^{+}$regulatory $\mathrm{T}$ cells to the site of inflammation along with other inflammatory leucocytes. The strong GFP signal indicating inflammation in the NOD islets, not observed in B6.Foxp3-GFP recipient mice (Fig. 4a), matched completely the reduced FOCI signal in the NOD islet (Fig. 4b). This supports the notion that this approach could be used to discriminate the beta cell volume from the infiltrated volume (Fig. 4b, ESM Fig. 4). The B6 control mice did not show such alterations in the FOCI signal. To confirm this, the mice were submitted to conventional IHC of the grafted islet. As illustrated in Fig. 4c and ESM Fig. 5, the insulin staining matched the brighter part of the segmented XfOCM image, while the significantly lower FOCI signal corresponded to the area stained with a pan-leucocyte marker. These findings confirm that FOCI can detect inflammation in addition to the beta cell volume. While the high OCM signal is specific to insulin, the lower OCM signal cannot be solely attributed to infiltration. Indeed, other non-beta endocrine cells and vessels also give weaker scattering. To estimate the extent to which the endocrine cell and vessel volume contribute to the non-beta cell volume in inflamed islets, we tried to quantify the nonbeta cell volume in non-inflamed healthy wild-type islets.
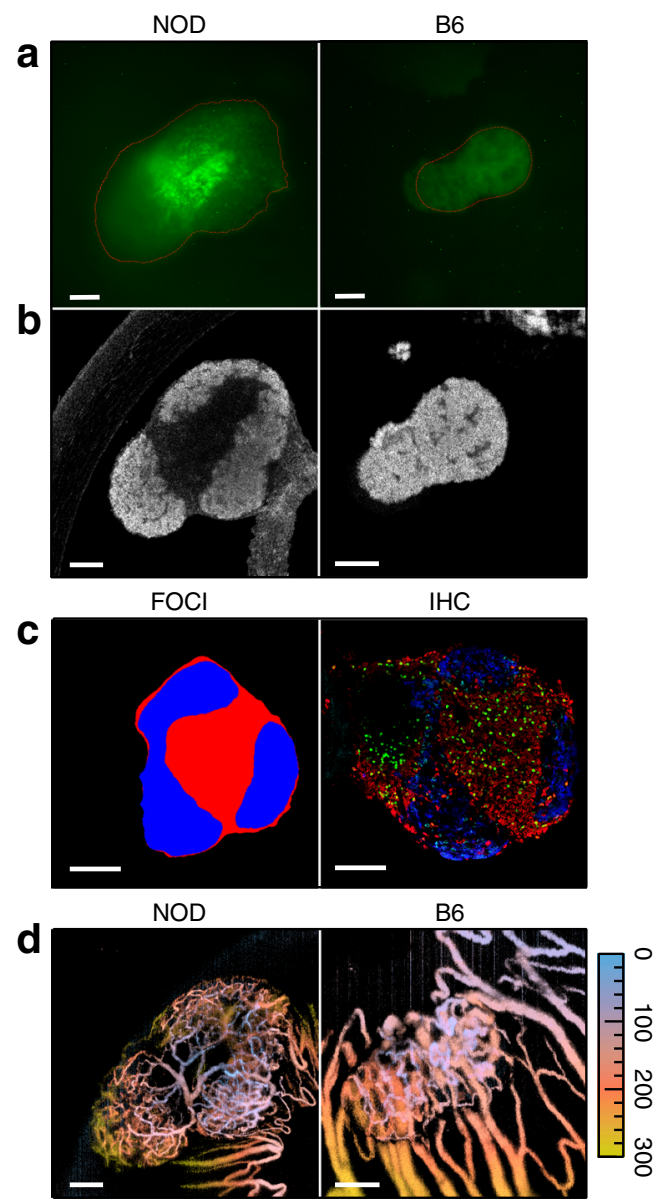

Fig. 4 FOCI detects inflammation in ACE-transplanted islets affected by insulitis. Islets transplanted into the ACE of 10-week-old NOD.Foxp3GFP recipients ( $n=12$ mice) with ongoing insulitis or healthy B6.Foxp3GFP control mice ( $n=2$ mice) were imaged by FOCI weekly. Shown is one representative imaging session at 4 weeks (NOD) or 5 weeks (B6) after transplantation. (a) Fluorescent widefield microscopy showing recruitment of FOXP3-GFP ${ }^{+}$T cells in NOD but not in B6 control mice. (b) FOCI recording showing structural information for the engrafted islet. (c) Structural FOCI section segmented for beta cell signal (blue) and inflammation (red) and cryosection (IHC) of the same graft-bearing eye $(n=7$ mice) at imaging endpoint stained with antibodies specific for insulin (blue) and CD45 (red), GFP signal (FOXP3 ${ }^{+} \mathrm{T}$ cells) shown in green. (d) Maximum projection of the vascularisation. Scale bar: $100 \mu \mathrm{m}$, colour bar indicates depth position in $\mu \mathrm{m}$

After initial fluctuations of the non-beta cell volume shortly after transplantation, it remained stable, making up approximately $3 \%$ of the total islet volume (ESM Fig. 6). While the mean value of the non-beta cell volume in the non-inflamed area is consistent with the literature [30], the significant deviation shows the limitation of FOCI technology for such a small volume.

To confirm that the insulitis identified in the ACEtransplanted NOD islets could be similarly detected by OCM in the pancreas, we next analysed images of surgically exposed pancreas from aged non-diabetic NOD mice. As illustrated in Fig. 5a-c, we observed alterations in the refractory 
Fig. 5 OCM imaging of mouse pancreas. Pancreas of prediabetic NOD.Foxp3-GFP mice $(n=6)(\mathbf{a}-$ c) or B6.Foxp3-GFP control mice $(n=2)$ (d) subjected to live xfOCM imaging after laparotomy. Maximum projection of vascularisation and the orthogonal view of islet structure in insulitis $(\mathbf{a}, \mathbf{b})$, peri-insulitis (c) and a non-inflamed control (d). Arrows indicate blood vessels, arrowheads indicate inflammation. Scale bar $100 \mu \mathrm{m}$, colour bar indicates depth position in $\mu \mathrm{m}$. Stripes in the vascularisation images are due to residual pancreas movements a
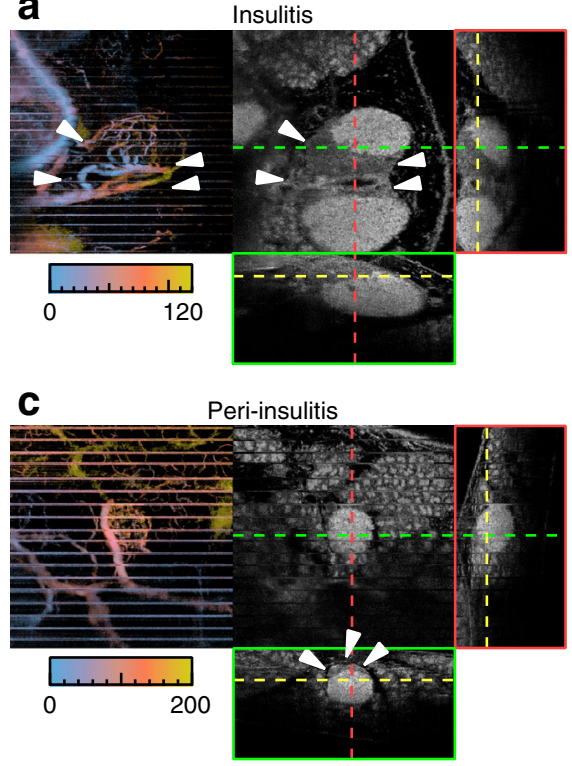

b

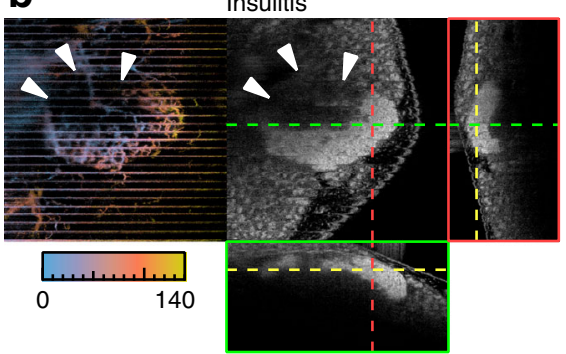

d

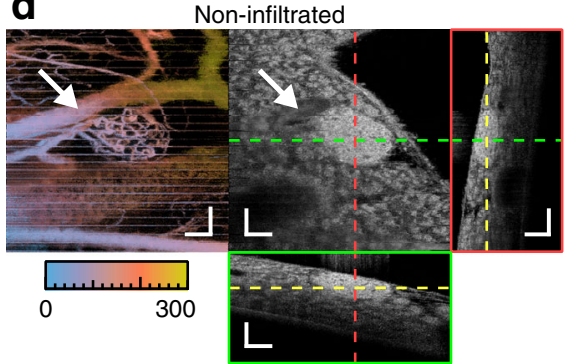

pattern in some of the in situ imaged NOD islets, but not in control B6 mice (Fig. 5d). This OCM signal was lower than the signal detected in beta cells and different from the signal detected from the exocrine tissue. These images potentially reflected insulitis spanning from unaffected to peri-islet infiltrates and full-blown intra-islet infiltration. By imaging thick pancreatic sections of NOD mice stained with a pan-leucocyte marker (Fig. 6), we further confirmed that, as in the ACE model, insulitis can be captured in situ by OCM in the pancreas, though with the limitation of getting only single snapshots of the disease.

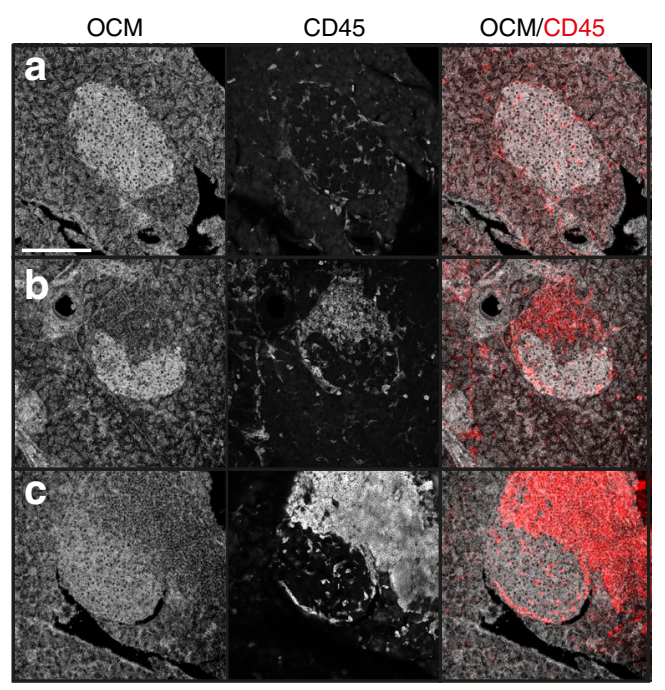

Fig. 6 Ex vivo OCM imaging of pancreas sections affected by insulitis. Pancreas sections of 16-week-old NOD.Foxp3-GFP mice $(n=3)$ were fixed and subjected to IHC using the pan-leucocyte marker CD45 and analysed by dfOCM and confocal microscopy (for imaging setup, see ESM Fig. 1). Representative images show peri-insulitis (a), mild (b) or massive insulitis (c). Scale bar: $100 \mu \mathrm{m}$
Insulitis correlates with attenuated vascularisation density Using the longitudinal FOCI platform, we next monitored the vascular network of the islets during progressive inflammation (Fig. 7). Ten-week-old recipient mice with an ongoing autoimmune process were used, anticipating that the insulitis in the ACE-transplanted islets of these mice would progress rapidly. As expected, Foxp3-GFP ${ }^{+}$cells were found to be recruited, along with other inflammatory cells, to the islet graft and accumulate over time (Fig. 7a), resulting in progressive beta cell destruction. This is clearly distinguishable in the orthogonal FOCI view (Fig. 7b), which is additionally illustrated in a 3D representation (Fig. 7c-e, ESM Video 1-3) and quantified (Fig. 7f).

As expected, we could visualise a gradual increase in the total islet vascular network shortly after transplantation (ESM Fig. 7). Counter intuitively, we noted a decrease in the microvasculature density in infiltrated regions of inflamed islets, with only a few big vessels remaining (Fig. 7a, g, h, Fig. 4d and ESM Videos 1-3). This decrease in the microvasculature density was not observed in islets displaying only limited infiltration (ESM Fig. 7 and ESM Video 4), indicating that the observed attenuation of microvasculature correlates with the severity of inflammation. Additionally, an attenuation of the microvascular network was observed in the inflamed islets imaged in situ in the surgically exposed pancreases (Fig. 5a-c), but not in control B6 mice in situ (Fig. 5d) or B6 islet grafts in the ACE (Fig. 2, Fig. 4d).

To confirm the regression of microvasculature observed by FOCI, we used IHC to analyse sections of pancreatic islets from aged non-diabetic NOD.Foxp3-GFP mice or ACEtransplanted islets from the same mice (4 weeks post transplantation) (see ESM Methods for details). This proves that 

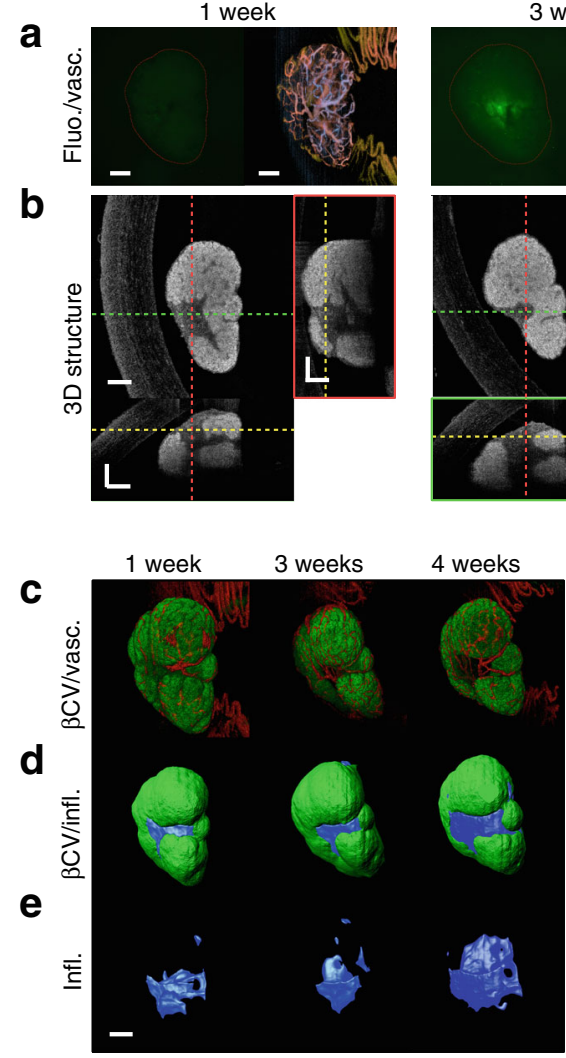

Fig. 7 Longitudinal quantification of insulitis and the impact on vascularisation. Islets, transplanted into the ACE of 10-week-old NOD.Foxp3-GFP reporter mice $(n=12)$ with ongoing insulitis, were imaged weekly. Shown are representative imaging sessions of the same islet at 1 week, 3 weeks and 4 weeks. (a) Live widefield fluorescence imaging and maximum projection of vascularisation. (b) Orthogonal view of a 3D stack of the islet graft at weeks 1-4. (c) Overlay of vasculature (red) and beta cell volume (green). (d) Segmented beta cell volume (green) and inflammation volume (blue) or (e) inflammation volume alone. (f)

the vessel density, as revealed by anti-CD31, was decreased in inflamed areas compared with non-affected areas of the islets (Fig. 8a-c, i). In contrast, high endothelial venules (HEVs) [31] expressing mucosal addressin cell adhesion molecule 1 (MAdCAM-1) predominated within inflamed islet areas (Fig. 8e-g, j). This supports previous observations of lymphocyte-HEV recognition in the development of insulitis in NOD mice $[8,15,32,33]$. Interestingly, a similar distribution of $\mathrm{CD} 31^{+}$vs HEVs was also seen in inflamed ACEtransplanted NOD islets (Fig. 8d, h, i, j).

\section{Discussion}

Our label-free 3D images are based on next-generation OCM, denoted functional optical coherence imaging for its substantial extension into functional imaging. Compared with related imaging techniques such as OCT [34]
3 weeks
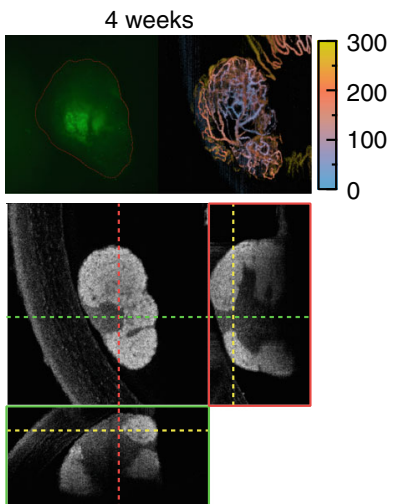

g

f

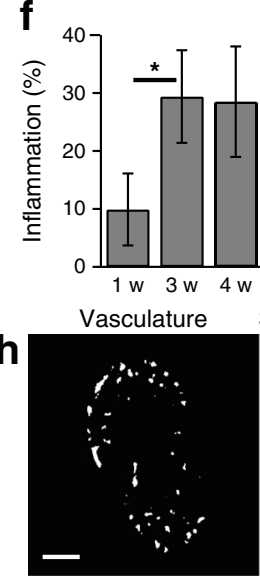

Quantification of inflammation volume per islet volume over time ( $n=$ 5). (g) Relative density of vascularisation in inflamed volumes of the islet (blue in d) compared with non-affected beta cell volume (green in d) at 4 weeks ( $n=5$ islets), see also ESM Methods. (h) FOCI section of vasculature alone or merged with islet structure. Scale bar: $100 \mu \mathrm{m}$, colourcoded bar indicates depth position in $\mu \mathrm{m} .{ }^{*} p<0.05$ and ${ }^{* *} p<0.01$, Mann-Whitney test. $\beta \mathrm{CV}$, beta cell volume; infl., inflammation; vasc., vasculature; $w$, week or optical frequency domain imaging (OFDI) [35], FOCI has a micrometric resolution over an extended depth range. In contrast to conventional confocal microscopy, FOCI offers several advantages for islet imaging. First, it allows for simultaneous, label-free imaging of the islet structure and the vasculature. As the FOCI contrast is caused by the intrinsic light scattering of tissue it does not require genetically modified mice or an extrinsic biomarker to detect the beta cell volume and functional vascularisation. In addition, it is not affected by autofluorescence. Second, the coherent amplification of the backscattered light results in a higher signal-to-noise ratio. This increased contrast enables quantitative segmentation of the beta cell volume, vascularisation and infiltration. Third, the multiplex advantage of FOCI translates into a parallel acquisition of depth profiles, circumventing the need for voxel per voxel scanning in depth. The fast acquisition increases the time resolution allowing for visualising the vasculature of 
NOD.Foxp3-GFP $14 \mathrm{w}$
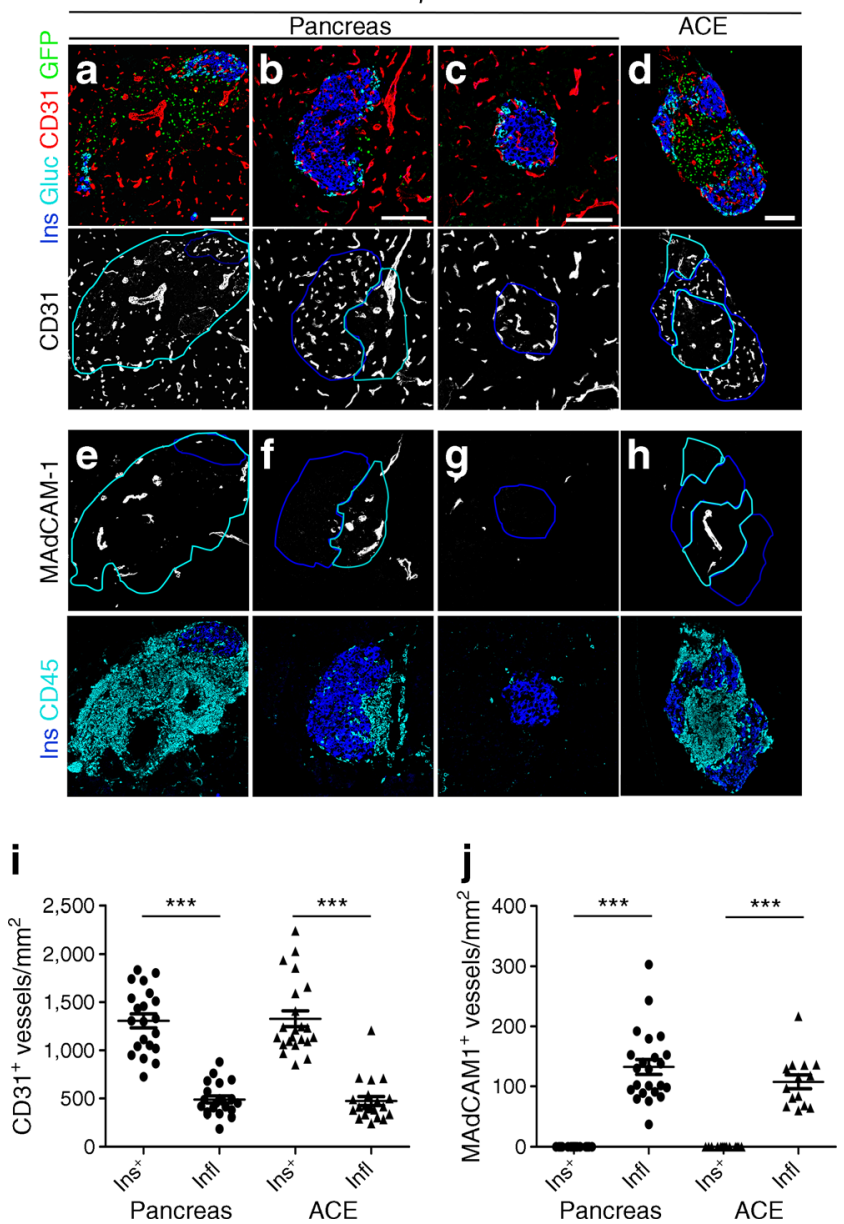

Fig. 8 Confirmation of the attenuation of microvasculature in insulitic areas by IHC and the identification of HEVs. IHC of transplanted eye and pancreas samples from 14-week-old NOD.Foxp3-GFP reporter mice $(n=$ 7) 4 weeks after transplantation, demonstrating the attenuated microvasculature by immunostaining for CD31 (a-d) and identification of HEVs by MAdCAM-1 staining (e-h). Beta cell areas were identified by insulin (blue line) and inflamed areas by the pan-leucocyte marker CD45 (cyan line) or DAPI. GFP signal (FOXP3 $3^{+}$T cells, a-d) is shown in green and glucagon staining in cyan (a-d). Representative images of the same NOD pancreas (a-c, e-g): unaffected (c, g); with mild insulitis $(\mathbf{b}, \mathbf{f})$; and with full-blown insulitis $(\mathbf{a}, \mathbf{e})$. Quantification of $\mathrm{CD} 1^{+}$(i) or MAdCAM- $1^{+}$ (j) vessel density (vessels $/ \mathrm{mm}^{2}$ ) in insulin-positive islet areas compared with inflamed islet areas $(n=13-23$ islet sections, 5-6 mice, see ESM Methods for further details). ${ }^{* *} p<0.001$; scale bars: $100 \mu \mathrm{m}$. w, weeks

whole islets in $30 \mathrm{~s}$. By applying the phase sensitivity of FOCI, blood flow can be quantified, a critical variable to monitor during the progression of diabetes. Finally, FOCI uses a broadband near infra-red (NIR) light source $(<5 \mathrm{~mW}$ on the cornea) to acquire a full depth, resulting in a substantially lower power exposure and minimises the risk of photo-damage of fragile islet structures and microvasculature.

The lack of direct, non-invasive technologies to visualise inflammation in the pancreas has been a key obstacle to the early detection of type 1 diabetes and the rapid assessment of the effectiveness of therapeutic intervention. To highlight the usefulness of FOCI applied to the ACE model, we analysed and compared islets from a mouse model of type 1 diabetes. The location and anatomy of the pancreas make the study of this disease at the organ level difficult, requiring compromises on resolution for longitudinal studies in the timescale of disease progression. While direct examination of fixed pancreases has yielded valuable insight into the processes leading to diabetes, such classic attempts only provide a snapshot of the disease. Thus, non-invasive imaging strategies to monitor changes within the islets associated with diabetes are being sought to fill this gap. By applying the FOCI technique, we revealed several surprising features of the disease.

The longitudinal recording of the autoimmune-induced islet alterations in the ACE provided detailed monitoring and quantification of the decreasing beta cell volume and of the impact on the islet vasculature during the inflammatory process. An observed decrease in the vascular density in areas affected by insulitis coincided with a promotion of MAdCAM-1-expressing HEV-like structures. Altered vasculature has previously been associated with type 1 diabetes $[2-4,36]$. More specifically, Villalta et al [36] have reported an increase rather than a decrease in vasculature when comparing pancreases from NOD mice prior to insulitis onset and NOD mice with advanced insulitis. This apparently conflicting statement may, in part, be due to differences in the overall assessment. We compared the vascularisation of infiltrated and non-infiltrated areas on the same individual islets, whereas Villalta et al [36] observed the increase in vasculature comparing the whole islets isolated from 4-week-old NOD mice with 18-weekold NOD mice. We therefore argue that the increase in vasculature under those conditions may be part of a developmentally controlled increase in islet vascularisation. HEVs have been linked to the promotion of guidance of lymphocyte trafficking and recruitment into inflammatory sites [31]. The observed overall reduction in the microvasculature is counterintuitive and remains to be understood in the larger framework of its impact on the pathogenesis of autoimmune diabetes.

The label-free detection of beta cell volume and vascularisation offered by the FOCI technique provides the unique possibility of studying ACE-transplanted human islets. Interestingly, the acceptance rate of xenografting human islets to NOD.Rag $2^{-/-}$mice was similar to that of grafting syngeneic NOD islets. This may be surprising, as recombination activating gene 2 (RAG2)-deficient mice have been shown to retain functional natural killer (NK) cells, which have been suggested to contribute to xenograft rejection [37]. However, the role of NK cells in the rejection of xenotransplanted solid organs remains debated [38] and we cannot fully explain the mechanisms. 
Acknowledgements We thank M. Sison (School of Engineering, Ecole Polytechnique Fédérale de Lausanne, Switzerland) for his help with the dfOCM setup.

Funding This work was partly financially supported by the Swiss National Science Foundation Grant (20320L-150191, 206021-139141), the Commission for Technology and Innovation (CTI, Bern, grant 17537.2 PFLS-LS), the Novo Nordisk Foundation, Diabetesförbundet, Barndiabetesförbundet and by the Swedish Research Council. D. Szlag acknowledges support from the Scientific Exchange Programme between Switzerland and the New Member States of the European Union and the project Enhancing Educational Potential of Nicolaus Copernicus University in the Disciplines of Mathematical and Natural Sciences grant.

Duality of interest The authors declare that there is no duality of interest associated with this manuscript.

Contribution statement $\mathrm{CB}, \mathrm{AS}-\mathrm{C}$, DS, JE, AG-B, TL and DH conceived and designed the experiments. AS-C, $\mathrm{LH}, \mathrm{CB}, \mathrm{JE}, \mathrm{AB}$ and $\mathrm{DS}$ performed, acquired and analysed the FOCI data. AS-C performed and analysed the immunohistochemistry experiments. DS implemented the vascularisation extraction. $\mathrm{CB}$ performed the analysis and quantification of the FOCI images. CB, JE, JG, MV, AG-B and FS performed, acquired and analysed the Znt8 and guinea pig experiments. CB, AS-C, DS, TL and $\mathrm{DH}$ drafted the manuscript. JE, LH, AB, MV, JG, FS and AG-B critically revised the manuscript. All authors approved the final version to be published and agreed to be accountable for all aspects of the work. $\mathrm{DH}$ is guarantor of this work.

\section{References}

1. Pober JS, Cotran RS (1990) The role of endothelial cells in inflammation. Transplantation 50:537-544

2. Papaccio G, Pisanti FA, Montefiano RD, Graziano A, Latronico MV (2002) Th1 and Th2 cytokines exert regulatory effects upon islet microvascular areas in the NOD mouse. J Cell Biochem 86: 651-664

3. Medarova $Z$, Greiner DL, Ifediba $M$ et al (2011) Imaging the pancreatic vasculature in diabetes models. Diabetes Metab Res Rev 27: 767-772

4. Medarova Z, Castillo G, Dai G, Bolotin E, Bogdanov A, Moore A (2007) Noninvasive magnetic resonance imaging of microvascular changes in type 1 diabetes. Diabetes 56:2677-2682

5. Dai C, Brissova M, Reinert RB et al (2013) Pancreatic islet vasculature adapts to insulin resistance through dilation and not angiogenesis. Diabetes 62:4144-4153

6. Carlsson PO, Sandler S, Jansson L (1998) Pancreatic islet blood perfusion in the nonobese diabetic mouse: diabetes-prone female mice exhibit a higher blood flow compared with male mice in the prediabetic phase. Endocrinology 139:3534-3541

7. Gaglia JL, Guimaraes AR, Harisinghani M et al (2011) Noninvasive imaging of pancreatic islet inflammation in type $1 \mathrm{~A}$ diabetes patients. J Clin Invest 121:442-445

8. Denis MC, Mahmood U, Benoist C, Mathis D, Weissleder R (2004) Imaging inflammation of the pancreatic islets in type 1 diabetes. Proc Natl Acad Sci U S A 101:12634-12639

9. Nyman LR, Wells KS, Head WS et al (2008) Real-time, multidimensional in vivo imaging used to investigate blood flow in mouse pancreatic islets. J Clin Invest 118:3790-3797

10. Lindsay RS, Corbin K, Mahne A et al (2015) Antigen recognition in the islets changes with progression of autoimmune islet infiltration. J Immunol 194:522-530
11. Coppieters K, Martinic MM, Kiosses WB, Amirian N, von Herrath $\mathrm{M}$ (2010) A novel technique for the in vivo imaging of autoimmune diabetes development in the pancreas by two-photon microscopy. Plos One 5

12. Coppieters K, Amirian N, von Herrath M (2012) Intravital imaging of CTLs killing islet cells in diabetic mice. J Clin Invest 122:119131

13. Alanentalo T, Asayesh A, Morrison $\mathrm{H}$ et al (2007) Tomographic molecular imaging and 3D quantification within adult mouse organs. Nat Methods 4:31-33

14. Alanentalo T, Loren CE, Larefalk A, Sharpe J, Holmberg D, Ahlgren U (2008) High-resolution three-dimensional imaging of islet-infiltrate interactions based on optical projection tomography assessments of the intact adult mouse pancreas. J Biomed Opt 13: 054070

15. Alanentalo T, Hornblad A, Mayans S et al (2010) Quantification and three-dimensional imaging of the insulitis-induced destruction of beta-cells in murine type 1 diabetes. Diabetes 59:1756-1764

16. Leitgeb RA, Villiger M, Bachmann AH, Steinmann L, Lasser T (2006) Extended focus depth for Fourier domain optical coherence microscopy. Opt Lett 31:2450-2452

17. Villiger M, Goulley J, Friedrich $M$ et al (2009) In vivo imaging of murine endocrine islets of Langerhans with extended-focus optical coherence microscopy. Diabetologia 52:1599-1607

18. Berclaz C, Pache C, Bouwens A et al (2015) Combined Optical Coherence and Fluorescence Microscopy to assess dynamics and specificity of pancreatic beta-cell tracers. Sci Rep 5:10385

19. Berclaz C, Goulley J, Villiger M et al (2012) Diabetes imagingquantitative assessment of islets of Langerhans distribution in murine pancreas using extended-focus optical coherence microscopy. Biomed Opt Express 3:1365-1380

20. Speier $\mathrm{S}$, Nyqvist $\mathrm{D}$, Cabrera $\mathrm{O}$ et al (2008) Noninvasive in vivo imaging of pancreatic islet cell biology. Nat Med 14:574-578

21. Schmidt-Christensen A, Hansen L, Ilegems E et al (2013) Imaging dynamics of $\mathrm{CD} 11 \mathrm{c}(+)$ cells and Foxp3(+) cells in progressive autoimmune insulitis in the NOD mouse model of type 1 diabetes. Diabetologia 56:2669-2678

22. Abdulreda MH, Faleo G, Molano RD et al (2011) High-resolution, noninvasive longitudinal live imaging of immune responses. Proc Natl Acad Sci U S A 108:12863-12868

23. Soderstrom I, Bergman ML, Colucci F, Lejon K, Bergqvist I, Holmberg D (1996) Establishment and characterization of RAG-2 deficient non-obese diabetic mice. Scand J Immunol 43:525-530

24. Lemaire K, Ravier MA, Schraenen A et al (2009) Insulin crystallization depends on zinc transporter ZnT8 expression, but is not required for normal glucose homeostasis in mice. Proc Natl Acad Sci U S A 106:14872-14877

25. Nyqvist D, Kohler M, Wahlstedt H, Berggren PO (2005) Donor islet endothelial cells participate in formation of functional vessels within pancreatic islet grafts. Diabetes 54:2287-2293

26. Villiger M, Pache C, Lasser T (2010) Dark-field optical coherence microscopy. Opt Lett 35:3489-3491

27. Lemaire K, Chimienti F, Schuit F (2012) Zinc transporters and their role in the pancreatic beta-cell. J Diabetes Investig 3:202-211

28. Conlon JM (2001) Evolution of the insulin molecule: insights into structure-activity and phylogenetic relationships. Peptides 22: $1183-1193$

29. Chmelova H, Cohrs CM, Chouinard JA et al (2015) Distinct roles of beta cell mass and function during type 1 diabetes onset and remission. Diabetes 64:2148-2160

30. Kim A, Miller K, Jo J, Kilimnik G, Wojcik P, Hara M (2009) Islet architecture: a comparative study. Islets 1:129-136

31. Mebius RE, Streeter PR, Michie S, Butcher EC, Weissman IL (1996) A developmental switch in lymphocyte homing receptor and endothelial vascular addressin expression regulates lymphocyte 
homing and permits CD4+ CD3- cells to colonize lymph nodes. Proc Natl Acad Sci U S A 93:11019-11024

32. Hanninen A, Taylor C, Streeter PR et al (1993) Vascular addressins are induced on islet vessels during insulitis in nonobese diabetic mice and are involved in lymphoid cell binding to islet endothelium. J Clin Invest 92:2509-2515

33. Hanninen A, Jaakkola I, Jalkanen S (1998) Mucosal addressin is required for the development of diabetes in nonobese diabetic mice. J Immunol 160:6018-6025

34. Huang D, Swanson EA, Lin CP et al (1991) Optical coherence tomography. Science 254:1178-1181
35. Yun S, Tearney G, de Boer J, Iftimia N, Bouma B (2003) Highspeed optical frequency-domain imaging. Opt Express 11:29532963

36. Villalta SA, Lang J, Kubeck S et al (2013) Inhibition of VEGFR-2 reverses type 1 diabetes in NOD mice by abrogating insulitis and restoring islet function. Diabetes 62:2870-2878

37. Chen D, Weber M, Lechler R, Dorling A (2006) NK-cell-dependent acute xenograft rejection in the mouse heart-to-rat model. Xenotransplantation 13:408-414

38. Griesemer A, Yamada K, Sykes M (2014) Xenotransplantation: immunological hurdles and progress toward tolerance. Immunol Rev 258:241-258 